Pacific

Journal of

Mathematics

\title{
LOCALLY LINEARLY DEPENDENT OPERATORS
}

Roy Meshulam and Peter Šemrl 


\title{
LOCALLY LINEARLY DEPENDENT OPERATORS
}

\author{
Roy Meshulam and Peter ŠEmrl
}

\begin{abstract}
Let $U$ and $V$ be vector spaces over a field $\mathrm{F}$. Linear operators $T_{1}, \ldots, T_{n}: U \rightarrow V$ are locally linearly dependent if $T_{1} u, \ldots, T_{n} u$ are linearly dependent for every $u \in U$. We extend and unify known results on locally linearly dependent operators and present two applications of these new results, one in algebra and one in functional analysis.
\end{abstract}

\section{Introduction.}

Let $T$ be a linear operator defined on a complex vector space $X$ and let $n$ be a positive integer. Kaplansky [4] proved that $T$ is algebraic of degree at most $n$ if and only if for every $x \in X$ the vectors $x, T x, \ldots, T^{n} x$ are linearly dependent. One consequence of Kaplansky's result is that if $X$ is a Banach space and $T: X \rightarrow X$ a bounded linear operator, then $T$ is algebraic if and only if for every $x \in X$ there exists a positive integer $n$ (depending on $x$ ) such that $x, T x, \ldots, T^{n} x$ are linearly dependent.

Let $U$ and $V$ be vector spaces over a field $\mathbb{F}$. Linear operators $T_{1}, \ldots, T_{n}$ : $U \rightarrow V$ are locally linearly dependent if $T_{1} u, \ldots, T_{n} u$ are linearly dependent for every $u \in U$. In view of Kaplansky's result it is natural to study the global consequences of local dependence. Amitsur [1] proved that for every $n$-tuple of locally linearly dependent operators $T_{1}, \ldots, T_{n}: U \rightarrow V$ there exist scalars $\alpha_{1}, \ldots, \alpha_{n}$, not all zero, such that $S=\alpha_{1} T_{1}+\ldots+\alpha_{n} T_{n}$ satisfies

$$
\operatorname{rank} S \leq\left(\begin{array}{c}
n+1 \\
2
\end{array}\right)-1
$$

Aupetit [2, p. 87] proved that if $U$ and $V$ are complex vector spaces then $S$ can be chosen so that

$$
\operatorname{rank} S \leq n-1 \text {. }
$$

It is easy to see that this estimate is sharp (see [3]). Brešar and Šemrl [3] extended Aupetit's result to the case where $\mathbb{F}$ is an arbitrary infinite field. We will show that the same conclusion holds also for finite fields.

For technical reasons it is sometimes convenient to deal with the linear spaces spanned by $n$-tuples of locally linearly dependent operators. For linear spaces $U$ and $V$ over a field $\mathbb{F}$ we denote by $\mathcal{L}(U, V)$ the set of all linear operators from $U$ into $V$. In case $U=V$ we write $\mathcal{L}(U)=\mathcal{L}(U, U)$. A subspace $\mathcal{S} \subset \mathcal{L}(U, V)$ is locally linearly dependent if for every $u \in U$ there 
exists a nonzero $S \in \mathcal{S}$ such that $S u=0$. In Section 2 we prove that if $\mathcal{S}$ is an $n$-dimensional locally linearly dependent subspace of $\mathcal{L}(U, V)$ then $\mathcal{S}$ contains a nonzero operator of rank at most $n-1$. When $\mathbb{F}$ has at least $n+2$ elements we give a stronger result: If $\mathcal{S}$ contains an operator of rank at least $n$, then $\mathcal{S}$ must contain a nonzero operator of rank at most $n-2$. We also show that if $\mathbb{F}$ is infinite and $\mathcal{S} \subset \mathcal{L}(U)$ is a locally linearly dependent $n$-dimensional space of pairwise commuting operators, then $\mathcal{S}$ contains a nonzero square-zero operator of rank at most $n-1$.

Let $X$ and $Y$ be (real or complex) Banach spaces. We denote by $\mathcal{B}(X, Y)$ and $\mathcal{B}_{F}(X, Y)$ the set of all linear bounded operators from $X$ into $Y$ and the set of all linear bounded finite rank operators from $X$ into $Y$, respectively. If $\mathcal{S} \subset \mathcal{B}(X, Y)$ is a linear subspace then we write $\mathcal{S}_{F}=\mathcal{S} \cap \mathcal{B}_{F}(X, Y)$ and we say that a linear subspace is nontrivial if it contains nonzero elements. Müller [7] extended the second part of Kaplansky's result on locally algebraic operators by proving that if $\mathcal{S} \subset \mathcal{B}(X, Y)$ is a locally linearly dependent space of countable dimension then $\mathcal{S}_{F}$ is nontrivial. Brešar and Šemrl [3] provided a short proof of this statement. Larson [5] proved that if $\mathcal{S} \subset \mathcal{B}(X, Y)$ is a locally linearly dependent subspace of countable dimension satisfying a certain "finite dimensional support" condition then $\mathcal{S}_{F}$ is nontrivial and locally linearly dependent. We will unify and extend these results.

It turns out that some of the results in Section 2 do not depend on the linearity of the space $\mathcal{S}$ but rather on $\mathcal{S}$ being an algebraic set. We briefly remark on these nonlinear extensions in Section 3.

We believe that problems concerning locally linearly dependent operators are interesting by themselves. However, they are also important because of applications. Amitsur studied them in order to obtain results on rings satisfying generalized polynomial identities [1]. Larson's motivation for studying them were problems concerning reflexivity and linear interpolation [5], while Brešar and Šemrl used the results on locally linearly dependent operators to characterize commuting pairs of continuous derivations $d, g$ of a Banach algebra $\mathcal{A}$ with the property that $(d g)(x)$ is quasi-nilpotent for every $x \in \mathcal{A}[\mathbf{3}]$. In the last section our new results will be applied to obtain an improvment of Amitsur's result on rings satisfying generalized polynomial identities and to get a shorter proof of the above mentioned result on derivations. Recall that a linear mapping $d: \mathcal{A} \rightarrow \mathcal{A}$ is called a derivation if $d(x y)=x d(y)+d(x) y$ for all $x, y \in \mathcal{A}$.

Let us fix the notation. If $A$ is any finite set then $|A|$ denotes its cardinality. For a nonzero vector $u$ belonging to a vector space $U$ we denote by $[u]$ the one-dimensional space spanned by $u$. Finally, $\mathbb{P} U=\{[u]: u \in U \backslash\{0\}\}$ denotes the projective space of $U$. 


\section{Locally linearly dependent operators.}

We begin this section with a statement which will help us reduce some problems concerning locally linearly dependent operators to the finite-dimensional case.

Proposition 2.1. Let $U$ and $V$ be vector spaces over a field $\mathbb{F}, \mathcal{S}$ an $n$-dimensional subspace of $\mathcal{L}(U, V)$, and $k$ a positive integer. Then there exists a positive integer $t \leq\left(\begin{array}{c}k+n-1 \\ k\end{array}\right)$ and rank $k$ idempotents $P_{1}, \ldots, P_{t} \in \mathcal{L}(U)$ and $Q_{1}, \ldots, Q_{t} \in \mathcal{L}(V)$ such that every $T \in \mathcal{S}$ of rank at least $k$ satisfies $\operatorname{rank}\left(Q_{i} T P_{i}\right)=k$ for some $i, 1 \leq i \leq t$.

Proof. Let $\left\{u_{\alpha}\right\}_{\alpha \in I}$ and $\left\{v_{\beta}\right\}_{\beta \in J}$ be fixed Hamel bases of $U$ and $V$, respectively. Operators in $\mathcal{L}(U, V)$ will be identified with matrices with respect to these two bases. To each subset $I_{0} \subset I$ with $\left|I_{0}\right|=k$ we can associate an idempotent whose range is $\operatorname{span}\left\{u_{\alpha}\right\}_{\alpha \in I_{0}}$ and whose null space is $\operatorname{span}\left\{u_{\alpha}\right\}_{\alpha \in I \backslash I_{0}}$. So, to prove our statement, it suffices to find subsets $I_{1}, \ldots, I_{t} \subset I, J_{1}, \ldots, J_{t} \subset J$, each of cardinality $k$, such that for every $T \in \mathcal{S}$ of rank at least $k$ we have $\operatorname{det} T\left[J_{i} \mid I_{i}\right] \neq 0$ for some $i, 1 \leq i \leq t$. Here $T\left[J_{i} \mid I_{i}\right]$ denotes the $k \times k$ submatrix of $T$ determined by $J_{i} \times I_{i}$.

The subsets $\left\{I_{i}\right\}_{i=1}^{t}$ and $\left\{J_{i}\right\}_{i=1}^{t}$ are constructed inductively as follows: Suppose $\left\{I_{i}\right\}_{i=1}^{l}$ and $\left\{J_{i}\right\}_{i=1}^{l}$ have already been chosen. If for any $T \in \mathcal{S}$ with $\operatorname{rank} T \geq k$ there exists an $i, 1 \leq i \leq l$, such that $\operatorname{det} T\left[J_{i} \mid I_{i}\right] \neq 0$ then let $t=l$. Otherwise there is an $T_{l+1} \in \mathcal{S}$ with rank at least $k$ such that $\operatorname{det} T_{l+1}\left[J_{i} \mid I_{i}\right]=0$ for all $i=1, \ldots, l$. We can find $I_{l+1} \subset I$ and $J_{l+1} \subset J$, both of cardinality $k$, such that $\operatorname{det} T_{l+1}\left[J_{l+1} \mid I_{l+1}\right] \neq 0$.

Let $S_{1}, \ldots, S_{n}$ be a basis of $\mathcal{S}$ and define for every positive integer $l \leq t$ (apriori $t$ might be infinite) a homogeneous polynomial $f_{l}$ of degree $k$ by

$$
f_{l}\left(x_{1}, \ldots, x_{n}\right)=\operatorname{det}\left(\sum_{i=1}^{n} x_{i} S_{i}\left[J_{l} \mid I_{l}\right]\right) .
$$

Let $a_{l}=\left(a_{l 1}, \ldots, a_{l n}\right)$ be coordinates of $T_{l}$ with respect to the basis $\left\{S_{1}, \ldots\right.$, $\left.S_{n}\right\}$, that is, $T_{l}=\sum_{i=1}^{n} a_{l i} S_{i}$. It follows from our inductive construction that $f_{l}\left(a_{l}\right) \neq 0,1 \leq l \leq t$, and $f_{l}\left(a_{l^{\prime}}\right)=0$ whenever $l^{\prime}>l$. Consequently, the polynomials $\left\{\bar{f}_{l}\right\}_{l=1}^{t}$ are linearly independent: If $\sum_{l=1}^{t} \lambda_{l} f_{l}=0$ is a nontrivial linear dependence and $l^{\prime}=\max \left\{l: \lambda_{l} \neq 0\right\}$ then

$$
0=\sum_{l=1}^{t} \lambda_{l} f_{l}\left(a_{l^{\prime}}\right)=\lambda_{l^{\prime}} f_{l^{\prime}}\left(a_{l^{\prime}}\right) \neq 0,
$$

a contradiction. Since the dimension of the space of all homogenous polynomials of degree $k$ in $n$ variables is $\left(\begin{array}{c}k+n-1 \\ k\end{array}\right)$, we have $t \leq\left(\begin{array}{c}k+n-1 \\ k\end{array}\right)$ as desired. 
We now prove that every $n$-dimensional locally linearly dependent space contains a nonzero operator of rank at most $n-1$. In fact, we will prove a slightly stronger result. An $n$-dimensional subspace $\mathcal{S} \subset \mathcal{L}(U, V)$ is locally linearly dependent if $\operatorname{dim} \mathcal{S} u=\operatorname{dim}\{S u: S \in \mathcal{S}\} \leq n-1$ for every $u \in U$. We say that $\mathcal{S} \subset \mathcal{L}(U, V)$ is c-locally linearly dependent if $\operatorname{dim} \mathcal{S} u \leq n-c$ for every $u \in U$.

Theorem 2.2. Let $U$ and $V$ be vector spaces over a field $\mathbb{F}$ and let $n, c$ be positive integers with $c \leq n-1$. If $\mathcal{S} \subset \mathcal{L}(U, V)$ is a c-locally linearly dependent subspace of dimension $n$ then there exists a nonzero $S \in \mathcal{S}$ such that $\operatorname{rank} S \leq n-c$.

Proof. In the case that $\mathbb{F}$ is an infinite field the proof is a direct adaptation of the argument for the $c=1$ case due to Brešar and Šemrl [3]. By induction we may assume that $\operatorname{dim} \mathcal{S} u=n-c$ for some $u \in U$. So, there exists $0 \neq S \in \mathcal{S}$ such that $S u=0$. We will complete the proof of this case by showing that the range of $S$ is contained in $\mathcal{S} u$. Suppose to the contrary that $S v \notin \mathcal{S} u$ for some $v \in U$. Let $\mathcal{S}_{1}$ be an $(n-c)$-dimensional subspace of $\mathcal{S}$ such that $\mathcal{S}_{1} u=\mathcal{S} u$. Then, by [3, Lemma 2.1] we can find a nonzero scalar $\alpha$ such that

$$
\operatorname{dim} \mathcal{S}_{1}(u+\alpha v)=n-c \quad \text { and } \quad S v \notin \mathcal{S}_{1}(u+\alpha v)
$$

Then $S(u+\alpha v)=\alpha S v \notin \mathcal{S}_{1}(u+\alpha v)$, and consequently, $\operatorname{dim} \mathcal{S}(u+\alpha v) \geq$ $n-c+1$, a contradiction.

Assume now that $\mathbb{F}$ is a finite field and $U$ is finite dimensional. Denote $|\mathbb{F}|=q$ and $\operatorname{dim} U=m$. Let

$$
Z=\{([u],[S]) \in \mathbb{P} U \times \mathbb{P S}: S u=0\} .
$$

On one hand, for every $u \in U$ we have $\operatorname{dim}\{S \in \mathcal{S}: S u=0\} \geq c$ hence

$$
|Z|=\sum_{[u] \in \mathbb{P} U}|\{[S]: S u=0\}| \geq|\mathbb{P} U| \frac{q^{c}-1}{q-1} .
$$

On the other hand, let $r=\min \{\operatorname{rank} S: S \in \mathcal{S} \backslash\{0\}\}$. Then the null space of every nonzero $S \in \mathcal{S}$ is at most $(m-r)$-dimensional therefore

$$
|Z|=\sum_{[S] \in \mathbb{P} \mathcal{S}}|\{[u]: S u=0\}| \leq|\mathbb{P S}| \frac{q^{m-r}-1}{q-1} .
$$

Comparing these two inequalities we obtain $\left(q^{m}-1\right)\left(q^{c}-1\right) \leq\left(q^{n}-1\right)\left(q^{m-r}-\right.$ $1)$. If $m=\operatorname{dim} U \leq n-c$ then $\operatorname{rank} S \leq n-c$ for all $S \in \mathcal{S}$. Otherwise 
$m \geq n-c+1$ hence

$$
\begin{aligned}
q^{m-r} & >q^{m-r}-1 \geq \frac{\left(q^{m}-1\right)\left(q^{c}-1\right)}{q^{n}-1} \\
& =\frac{\left(q^{m}-1\right)\left(q^{c}-1\right)}{q^{m+c-1}-1} \frac{q^{m+c-1}-1}{q^{n}-1} \\
& \geq \frac{\left(q^{m}-1\right)\left(q^{c}-1\right)}{q^{m+c-1}-1} q^{m+c-n-1} \geq(q-1) q^{m+c-n-1} \geq q^{m+c-n-1} .
\end{aligned}
$$

It follows that $r \leq n-c$.

It remains to consider the case that $\mathbb{F}$ is a finite field and $U$ an infinite dimensional vector space. Assume that $\operatorname{rank} S \geq n-c+1$ for all nonzero operators $S \in \mathcal{S}$. Applying Proposition 2.1 with $k=n-c+1$ we obtain finitely many idempotents $P_{1}, \ldots, P_{t} \in \mathcal{L}(U)$ of rank $n-c+1$ such that for every nonzero $S \in \mathcal{S}$ there exists an $i, 1 \leq i \leq t$, such that rank $S P_{i}=$ $n-c+1$. Let $U^{\prime}=\sum_{i=1}^{t} P_{i} U$ and for $S \in \mathcal{S}$ let $S^{\prime} \in \mathcal{L}\left(U^{\prime}, V\right)$ denote the restriction of $S$ to $U^{\prime}$. It follows that $\mathcal{S}^{\prime}=\left\{S^{\prime}: S \in \mathcal{S}\right\}$ is an $n$-dimensional $c$-locally linearly dependent subspace of $\mathcal{L}\left(U^{\prime}, V\right)$ and $\operatorname{rank} S^{\prime} \geq n-c+1$ for all nonzero $S^{\prime} \in \mathcal{S}^{\prime}$, contradicting the finite-dimensional case.

For a subspace $\mathcal{S} \subset \mathcal{L}(U, V)$ we denote by $\mathcal{S}_{F}$ the space of all finite rank operators in $\mathcal{S}$. Larson [5, Proposition 2.3] proved that if $\mathcal{S}$ is finite dimensional locally linearly dependent, then $\mathcal{S}_{F}$ is a nontrivial locally linearly dependent subspace. Combining this with Theorem 2.2 we get the following result.

Corollary 2.3. Let $U$ and $V$ be vector spaces over a field $\mathbb{F}$. If $\mathcal{S} \subset \mathcal{L}(U, V)$ is a locally linearly dependent finite dimensional subspace then there exists a nonzero $S \in \mathcal{S}$ such that $\operatorname{rank} S \leq \operatorname{dim} \mathcal{S}_{F}-1$.

Theorem 2.2 can be improved for sufficiently large fields. For simplicity we only consider the $c=1$ case.

Theorem 2.4. Let $n$ be a positive integer and let $\mathbb{F}$ be a field with at least $n+2$ elements. Suppose that $U$ and $V$ are vector spaces over $\mathbb{F}$ and $\mathcal{S} \subset$ $\mathcal{L}(U, V)$ is an $n$-dimensional locally linearly dependent space of operators. If $\mathcal{S}$ contains an operator of rank at least $n$ then there is a nonzero $S \in \mathcal{S}$ such that $\operatorname{rank} S \leq n-2$.

Proof. By an application of Proposition 2.1 we may assume that $U$ and $V$ are finite dimensional. We will identify $U$ with $\mathbb{F}^{m}, V$ with $\mathbb{F}^{p}$, and $\mathcal{L}(U, V)$ with $M_{p \times m}(\mathbb{F})$, the space of all $p \times m$ matrices. There is no loss of generality in assuming that $m>n$.

To illustrate our approach we first give a simple proof of the theorem for an algebraically closed field $\mathbb{F}$. Consider the projective algebraic variety

$$
Z=\{([u],[S]) \in \mathbb{P} U \times \mathbb{P} \mathcal{S}: S u=0\}
$$


and let $\pi_{1}, \pi_{2}$ denote the projections of $Z$ into $\mathbb{P} U$ and $\mathbb{P} \mathcal{S}$ respectively. Since $\mathcal{S}$ is locally linearly dependent the projection $\pi_{1}$ is surjective, hence

$$
\operatorname{dim} Z \geq \operatorname{dim} \mathbb{P} U \text {. }
$$

Let $Z_{0}$ be an irreducible component of $Z$ of maximal dimension then

$$
\begin{aligned}
\operatorname{dim} Z & =\operatorname{dim} Z_{0}=\operatorname{dim} \pi_{2}\left(Z_{0}\right)+\min \left\{\operatorname{dim} \pi_{2}^{-1}([S]):[S] \in \pi_{2}\left(Z_{0}\right)\right\} \\
& =\operatorname{dim} \pi_{2}\left(Z_{0}\right)+\operatorname{dim} \mathbb{P} U-\max \left\{\operatorname{rank} S:[S] \in \pi_{2}\left(Z_{0}\right)\right\} .
\end{aligned}
$$

Combining (1) and (2) we obtain

$$
\max \left\{\operatorname{rank} S:[S] \in \pi_{2}\left(Z_{0}\right)\right\} \leq \operatorname{dim} \pi_{2}\left(Z_{0}\right) .
$$

If $\pi_{2}\left(Z_{0}\right)=\mathbb{P} \mathcal{S}$ then $\max \{\operatorname{rank} S: S \in \mathcal{S}\} \leq \operatorname{dim} \mathbb{P} \mathcal{S}=n-1$, contradicting our assumptions. Therefore, $\pi_{2}\left(Z_{0}\right) \neq \mathbb{P} \mathcal{S}$, hence

$$
\max \left\{\operatorname{rank} S:[S] \in \pi_{2}\left(Z_{0}\right)\right\} \leq \operatorname{dim} \pi_{2}\left(Z_{0}\right) \leq n-2 .
$$

The proof of Theorem 2.4 for general infinite fields uses a similiar idea: We will show that if $\mathcal{S}$ contains an operator of rank $\geq n$ then both $U_{1}=$ $\bigcup_{\text {rank } S \geq n} \mathbb{P}(\operatorname{ker} S)$ and $U_{2}=\bigcup_{\text {rank } S=n-1} \mathbb{P}(\operatorname{ker} S)$ are contained in proper algebraic subsets of $\mathbb{P} U$. Since $\mathbb{F}$ is infinite it will follow that there exists a $[u] \in \mathbb{P} U-\left(U_{1} \cup U_{2}\right)$. Let $0 \neq S \in \mathcal{S}$ such that $S u=0$ then $\operatorname{rank} S \leq n-2$. We need some preliminaries.

Claim 1. Let $x=\left(x_{1}, \ldots, x_{n}\right), y=\left(y_{1}, \ldots, y_{m-n}\right)$ be variables and let $f_{\text {st }}(x), 1 \leq s \leq m, 1 \leq t \leq m-n$, be homogeneous polynomials of the same degree. Then there exists a nonzero homogeneous polynomial $P\left(z_{1}, \ldots, z_{m}\right)$ such that

$$
P\left(\sum_{t=1}^{m-n} f_{1 t}(x) y_{t}, \ldots, \sum_{t=1}^{m-n} f_{m t}(x) y_{t}\right)=0 .
$$

Proof. Introduce new variables $\xi=\left(\xi_{1}, \ldots, \xi_{m-n-1}\right)$ and for $1 \leq i \leq m$ let $F_{i}(x, y)=\sum_{t=1}^{m-n} f_{i t}(x) y_{t}, G_{i}(x, \xi)=\sum_{t=1}^{m-n-1} f_{i t}(x) \xi_{t}+f_{i, m-n}(x)$. Since $G_{1}(x, \xi), \ldots, G_{m}(x, \xi)$ are polynomials in $m-1$ variables there exists a nonzero polynomial $R\left(z_{1}, \ldots, z_{m}\right)=\sum_{\gamma} r_{\gamma} z^{\gamma}$ (where $\gamma=\left(\gamma_{1}, \ldots, \gamma_{m}\right)$, $\left.z^{\gamma}=z_{1}^{\gamma_{1}} \cdots z_{m}^{\gamma_{m}}\right)$ such that $R\left(G_{1}(x, \xi), \ldots, G_{m}(x, \xi)\right)=0$. Write $|\gamma|=$ $\sum_{i=1}^{m} \gamma_{i}$ and let $N=\max \left\{|\gamma|: r_{\gamma} \neq 0\right\}$. By substituting $\xi_{i}=y_{i} / y_{m-n}$ for $1 \leq i \leq m-n-1$ it is easy to check that $P\left(z_{1}, \ldots, z_{m}\right)=\sum_{|\gamma|=N} r_{\gamma} z^{\gamma}$ satisfies $P\left(F_{1}(x, y), \ldots, F_{m}(x, y)\right)=0$.

Claim 2. Let $x=\left(x_{1}, \ldots, x_{n}\right), y=\left(y_{1}, \ldots, y_{m-n+1}\right)$ be variables and let $f_{\text {st }}(x), 1 \leq s \leq m, 1 \leq t \leq m-n+1$, be homogeneous polynomials of the same degree. Then for any nonzero homogenous polynomial $g(x)$ there 
exists a nonzero homogeneous polynomial $Q(z) \in \mathbb{F}\left[z_{1}, \ldots, z_{m}\right]$ such that

$$
Q\left(\sum_{t=1}^{m-n+1} f_{1 t}(a) y_{t}, \ldots, \sum_{t=1}^{m-n+1} f_{m t}(a) y_{t}\right)=0
$$

for any $a \in \mathbb{F}^{n}$ which satisfies $g(a)=0$.

Proof. Introduce new variables $\xi=\left(\xi_{1}, \ldots, \xi_{m-n}\right)$ and for $1 \leq i \leq m$ let $F_{i}(x, y)=\sum_{t=1}^{m-n+1} f_{i t}(x) y_{t}, G_{i}(x, \xi)=\sum_{t=1}^{m-n} f_{i t}(x) \xi_{t}+f_{i, m-n+1}(x)$. Since $G_{1}(x, \xi), \ldots, G_{m}(x, \xi), g(x)$ are $m+1$ polynomials in $m$ variables there exist polynomials $Q_{0}(z), \ldots, Q_{k}(z) \in \mathbb{F}\left[z_{1}, \ldots, z_{m}\right]$, not all of them zero, such that $\sum_{i=0}^{k} Q_{i}\left(G_{1}(x, \xi), \ldots, G_{m}(x, \xi)\right) g(x)^{i}=0$. Since $g(x)$ is homogenous and nonzero we may assume that all the $Q_{i}$ 's are homogenous and $Q_{0}(z) \neq$ 0 . As in Claim 1 it can be checked that $Q_{0}\left(F_{1}(a, y), \ldots, F_{m}(a, y)\right)=0$ whenever $g(a)=0$.

For an integer $t, 1 \leq t \leq \min \{p, m\}$, we define $A_{t}=\{(I, J): I \subset$ $\{1, \ldots, p\}, J \subset\{1, \ldots, m\},|I|=|J|=t\}$. Let $S_{1}, \ldots, S_{n}$ be a basis of $\mathcal{S}$ and denote $S(x)=S\left(x_{1}, \ldots, x_{n}\right)=\sum_{i=1}^{n} x_{i} S_{i}$.

Claim 3. For any $(I, J) \in A_{n}$ there exists a nonzero homogeneous form $P_{I J}(z) \in \mathbb{F}\left[z_{1}, \ldots, z_{m}\right]$ such that if $a \in \mathbb{F}^{n}$ and $u \in \mathbb{F}^{m}$ satisfy $S(a) u=0$ then

$$
P_{I J}(\lambda u)=0
$$

where $\lambda=\operatorname{det} S(a)[I \mid J]$.

Proof. We may assume that $I=\{1, \ldots, n\}, J=\{m-n+1, \ldots, m\}$. By Cramer's rule for any $m-n+1 \leq i \leq m$ there exist homogenous polynomials $f_{i j}(x), 1 \leq j \leq m-n$, of degree $n$ such that for any $a \in \mathbb{F}^{n}$ and $u \in \operatorname{ker} S(a)$

$$
\operatorname{det} S(a)[I \mid J] u_{i}=\sum_{j=1}^{m-n} f_{i j}(a) u_{j}
$$

By defining $f_{i j}(x)=\delta_{i j} \operatorname{det} S(x)[I \mid J]$ for $1 \leq i, j \leq m-n$, Equation (6) becomes valid for all $1 \leq i \leq m$. According to Claim 1 there exists a nonzero homogenous polynomial $P\left(z_{1}, \ldots, z_{m}\right)$ which satisfies (3). Combining (6) and (3) it follows that $P(\operatorname{det} S(a)[I \mid J] u)=0$ whenever $S(a) u=0$.

Claim 4. For every nonzero homogenous polynomial $g(x) \in \mathbb{F}\left[x_{1}, \ldots, x_{n}\right]$ and every $\left(I^{\prime}, J^{\prime}\right) \in A_{n-1}$ there exists a nonzero homogeneous form $Q_{I^{\prime} J^{\prime}}(z)$ $\in \mathbb{F}\left[z_{1}, \ldots, z_{m}\right]$ such that if $a \in \mathbb{F}^{n}, u \in \mathbb{F}^{m}$ satisfy $g(a)=0$ and $S(a) u=0$ then

$$
Q_{I^{\prime} J^{\prime}}(\lambda u)=0
$$

where $\lambda=\operatorname{det} S(a)\left[I^{\prime} \mid J^{\prime}\right]$. 
Proof. Let $I^{\prime}=\{1, \ldots, n-1\}, J^{\prime}=\{m-n+2, \ldots, m\}$. As in Claim 3 there exist homogenous polynomials $f_{i j}(x), 1 \leq i \leq m, 1 \leq j \leq m-n+1$ of degree $n-1$ such that if $a \in \mathbb{F}^{n}, u \in \mathbb{F}^{m}$ satisfy $S(a) u=0$ then for all $1 \leq i \leq m$

$$
\operatorname{det} S(a)\left[I^{\prime} \mid J^{\prime}\right] u_{i}=\sum_{j=1}^{m-n+1} f_{i j}(a) u_{j} .
$$

By Claim 2 there exists a homogenous polynomial $Q\left(z_{1}, \ldots, z_{m}\right)$ which satisfies (4) if $g(a)=0$. Combining (8) and (4) it follows that $Q\left(\operatorname{det} S(a)\left[I^{\prime} \mid J^{\prime}\right] u\right)$ $=0$ whenever $S(a) u=0$ and $g(a)=0$.

Now we are ready to prove the Theorem for infinite fields. Let $\left\{P_{I J}(z)\right.$ : $\left.(I, J) \in A_{n}\right\}$ be the forms determined by Claim 3. Suppose that rank $S_{0} \geq n$ for some $S_{0} \in \mathcal{S}$. Then there exists an $\left(I_{0}, J_{0}\right) \in A_{n}$ such that rank $S_{0}\left[I_{0} \mid J_{0}\right]$ $=n$. Let $g(x)=\operatorname{det}\left(S(x)\left[I_{0} \mid J_{0}\right]\right)$. Clearly, $g(x)$ is a nonzero homogenous form in $\mathbb{F}\left[x_{1}, \ldots, x_{n}\right]$. Let $\left\{Q_{I^{\prime} J^{\prime}}(z):\left(I^{\prime}, J^{\prime}\right) \in A_{n-1}\right\}$ be the forms determined by Claim 4 and the above $g(x)$. For any homogenous form $R(z) \in \mathbb{F}\left[z_{1}, \ldots, z_{m}\right]$ let $V(R)=\{[u] \in \mathbb{P} U: R(u)=0\}$ denote the projective zero set of $R(z)$.

Let $0 \neq a \in \mathbb{F}^{n}$. If $\operatorname{rank} S(a) \geq n$ then there exists an $(I, J) \in A_{n}$ such that $\operatorname{det}(S(a)[I \mid J]) \neq 0$. Hence, by $(5), P_{I J}(u)=0$ for any $u$ which satisfies $S(a) u=0$. It follows that

$$
\bigcup_{\left\{a \in \mathbb{F}^{n}: \operatorname{rank} S(a) \geq n\right\}} \mathbb{P}(\operatorname{ker} S(a)) \subset \bigcup_{(I, J) \in A_{n}} V\left(P_{I J}\right) .
$$

If on the other hand $\operatorname{rank} S(a)=n-1$ then there exists an $\left(I^{\prime}, J^{\prime}\right) \in$ $A_{n-1}$ such that $\operatorname{det}\left(S(a)\left[I^{\prime} \mid J^{\prime}\right]\right) \neq 0$. Since $g(a)=0$ it follows by $(7)$ that $Q_{I^{\prime} J^{\prime}}(u)=0$ for any $u \in \operatorname{ker} S(a)$. Therefore

$$
\bigcup_{\left\{a \in \mathbb{F}^{n}: \operatorname{rank} S(a)=n-1\right\}} \mathbb{P}(\operatorname{ker} S(a)) \subset \bigcup_{\left(I^{\prime}, J^{\prime}\right) \in A_{n-1}} V\left(Q_{I^{\prime} J^{\prime}}\right) .
$$

Let

$$
R(z)=\prod_{(I, J) \in A_{n}} P_{I J}(z) \prod_{\left(I^{\prime}, J^{\prime}\right) \in A_{n-1}} Q_{I^{\prime} J^{\prime}}(z) .
$$

Combining (9) and (10) we obtain

$$
\bigcup_{\left\{a \in \mathbb{F}^{n}: \operatorname{rank} S(a) \geq n-1\right\}} \mathbb{P}(\operatorname{ker} S(a)) \subset V(R) .
$$

Since $R(z)$ is a nonzero homogenous form and $\mathbb{F}$ is infinite there exists a $[u] \in \mathbb{P} U \backslash V(R)$. Let $0 \neq a \in \mathbb{F}^{n}$ be an $n$-tuple such that $S(a) u=0$. Then $\operatorname{rank} S(a) \leq n-2$. 
It remains to consider the finite field case. Let $\mathbb{F}$ be the field with $q$ elements, $q \geq n+2$. Let

$$
Z=\left\{([u],[a]) \in \mathbb{P} U \times \mathbb{P F}^{n}: S(a) u=0\right\}
$$

and suppose $a_{0} \in \mathbb{F}^{n}$ satisfies $\operatorname{rank} S\left(a_{0}\right) \geq n$. Since $\mathcal{S}$ is locally linearly dependent we have

$$
|Z|=\sum_{[u] \in \mathbb{P} U}|\{[a]: S(a) u=0\}| \geq|\mathbb{P} U|=\frac{q^{m}-1}{q-1} .
$$

Let $r=\min \{\operatorname{rank} S: S \in \mathcal{S} \backslash\{0\}\}$, let $\left(I_{0}, J_{0}\right) \in A_{n}$ be such that $\operatorname{rank} S\left(a_{0}\right)\left[I_{0} \mid J_{0}\right]=n$, and let $g(x)=\operatorname{det}\left(S(x)\left[I_{0} \mid J_{0}\right]\right)$. Clearly, if $a \in \mathbb{F}^{n}$ satisfies $\operatorname{rank} S(a) \leq n-1$ then $g(a)=0$. Therefore

$$
\begin{gathered}
|Z|=\sum_{[a] \in \mathbb{P}^{n}}|\mathbb{P}(\operatorname{ker} S(a))|= \\
\sum_{\{[a]: \operatorname{rank} S(a) \leq n-1\}}|\mathbb{P}(\operatorname{ker} S(a))|+\sum_{\{[a]: \operatorname{rank} S(a) \geq n\}}|\mathbb{P}(\operatorname{ker} S(a))| \leq \\
|\{[a]: g(a)=0\}| \frac{q^{m-r}-1}{q-1}+\left|\mathbb{P} \mathbb{F}^{n}\right| \frac{q^{m-n}-1}{q-1} .
\end{gathered}
$$

We need the following simple upper bound (see e.g., Theorem 6.15 in [6]).

Proposition 2.5. Assume that $|\mathbb{F}|=q$. If $h\left(x_{1}, \ldots, x_{k}\right) \in \mathbb{F}\left[x_{1}, \ldots, x_{k}\right]$ is a nonzero homogenous form of degree $d^{\prime} \geq 1$ then

$$
\left|\left\{[a] \in \mathbb{P F}^{k}: h(a)=0\right\}\right| \leq d^{\prime} \frac{q^{k-1}-1}{q-1} .
$$

Now $g(x)$ is homogenous of degree $n$ and satisfies $g\left(a_{0}\right) \neq 0$. Combining Proposition 2.5 (with $h=g, k=n$ and $d^{\prime}=n$ ) with the previous inequalities we obtain

$$
\frac{q^{m}-1}{q-1} \leq|Z| \leq n \frac{q^{n-1}-1}{q-1} \frac{q^{m-r}-1}{q-1}+\frac{q^{n}-1}{q-1} \frac{q^{m-n}-1}{q-1} .
$$

It follows that

$$
\begin{gathered}
q^{m-r}-1 \geq \frac{\left(q^{m}-1\right)(q-1)-\left(q^{n}-1\right)\left(q^{m-n}-1\right)}{n\left(q^{n-1}-1\right)} \geq \\
\frac{q^{m+1}-2 q^{m}}{n q^{n-1}}=\frac{q^{m-n+1}(q-2)}{n} \geq q^{m-n+1}
\end{gathered}
$$

hence $r \leq n-2$.

Theorem 2.2 with $c=1$ can be improved also in the case that $\mathbb{F}$ is an infinite field and $\mathcal{S}$ consists of pairwise commuting matrices. 
Theorem 2.6. Let $U$ be a vector space over an infinite field $\mathbb{F}$ and let $n$ be a positive integer. If $\mathcal{S} \subset \mathcal{L}(U)$ is an $n$-dimensional locally linearly dependent subspace of pairwise commuting matrices then there exists a nonzero $S \in \mathcal{S}$ such that $S^{2}=0$ and $\operatorname{rank} S \leq n-1$.

Remarks. The special case when $\mathbb{F}$ is the field of complex numbers was proved in [2, p. 87]. This result is important for applications (see the next section). In fact, we will need, and therefore we will prove, a slightly stronger statement as follows: Assume that $S_{1}, \ldots, S_{n}: U \rightarrow U$ are locally linearly dependent operators satisfying $S_{i} S_{j}=S_{j} S_{i}, 1 \leq i, j \leq n$. Suppose also that $S_{1}, \ldots, S_{n-1}$ are not locally linearly dependent. Then there exists scalars $\alpha_{1}, \ldots, \alpha_{n-1}$ such that $S=\alpha_{1} S_{1}+\ldots+\alpha_{n-1} S_{n-1}+S_{n}$ is of square zero and $\operatorname{rank} S \leq n-1$.

Proof. We can find $u \in U$ such that $S_{1} u, \ldots, S_{n-1} u$ are linearly independent. Then there exist scalars $\alpha_{1}, \ldots, \alpha_{n-1}$ such that $S u=\left(\alpha_{1} S_{1}+\right.$ $\left.\ldots \alpha_{n-1} S_{n-1}+S_{n}\right) u=0$. As in the proof of Theorem 2.2 we show that the range of $\mathcal{S}$ is contained in the linear span of $S_{1} u, \ldots, S_{n-1} u$. Let $v \in U$ be any vector. Then there exist $\beta_{1}, \ldots, \beta_{n-1} \in \mathbb{F}$ such that $S v=$ $\beta_{1} S_{1} u+\ldots+\beta_{n-1} S_{n-1} u$. It follows that $S^{2} v=S\left(\beta_{1} S_{1}+\ldots+\beta_{n-1} S_{n-1}\right) u=$ $\left(\beta_{1} S_{1}+\ldots+\beta_{n-1} S_{n-1}\right) S u=0$. This completes the proof.

We will conclude this section by unifying and extending the results of Larson [5, Theorem 3.2] and Müller [7, Theorem 1].

Theorem 2.7. Let $X$ and $Y$ be Banach spaces and let $\mathcal{S} \subset \mathcal{B}(X, Y)$ be a locally linearly dependent linear space of countable dimension. Then $\mathcal{S}_{F}$ has a nontrivial finite dimensional locally linearly dependent subspace.

Remark. Larson obtained this result under an additional "finite dimensional support" assumption on $\mathcal{S}_{F}$. The above theorem is also an essential improvment of Müller's result. Namely, the conclusion of the result of Müller is that $\mathcal{S}$ contains a nonzero finite rank operator. His result gives no information how large is the space $\mathcal{S}_{F}$ nor what is the minimal rank of nonzero operators from $\mathcal{S}$. Our result implies that either $\mathcal{S}$ contains an operator of a small rank or the dimension of $\mathcal{S}_{F}$ is large. More precisely, if the minimal nonzero rank of operators from $\mathcal{S}$ is $n$, then $\mathcal{S}_{F}$ is at least $(n+1)$-dimensional. This follows directly from Theorems 2.2 and 2.7 .

Proof. Let $\left\{S_{k}: k=1,2, \ldots\right\}$ be a basis of $\mathcal{S}$ and denote by $\mathcal{F}_{n}$ the set of all vectors $x \in X$ such that $S_{1} x, \ldots, S_{n} x$ are linearly dependent. Then $\cup_{n=1}^{\infty} \mathcal{F}_{n}=X$.

If $\left\{x_{m}\right\}$ is a sequence in $\mathcal{F}_{n}$ converging to $x$, then $x \in \mathcal{F}_{n}$. For suppose that for each $m, \alpha_{1}^{m}, \ldots, \alpha_{n}^{m}$ are scalars, not all zero, such that $\alpha_{1}^{m} S_{1} x_{m}+$ $\ldots+\alpha_{n}^{m} S_{n} x_{m}=0$. Divide these scalars by the one with the largest absolute 
value. We can thus assume that each one of them has absolute value at most 1 and one of them has absolute value equal to 1 . Then a subsequence of the sequence of $n$-tuples $\left\{\left(\alpha_{1}^{m}, \ldots, \alpha_{n}^{m}\right)\right\}$ converges to an $n$-tuple $\left(\alpha_{1}, \ldots, \alpha_{n}\right)$; it is not the zero $n$-tuple since at least one of $\alpha_{1}, \ldots, \alpha_{n}$ has modulus 1 . Re-label so that $\left\{\left(\alpha_{1}^{m}, \ldots, \alpha_{n}^{m}\right)\right\}$ is such a subsequence. Then $\alpha_{1} S_{1} x+\ldots+$ $\alpha_{n} S_{n} x=\lim _{m \rightarrow \infty}\left(\alpha_{1}^{m} S_{1} x_{m}+\cdots+\alpha_{n}^{m} S_{n} x_{m}\right)=0$.

Thus, each $\mathcal{F}_{n}$ is closed and by the Baire category theorem there exists a positive integer $k$ such that $\mathcal{F}_{k}$ contains an open ball, say $\{x \in X$ : $\left.\left\|x-x_{0}\right\|<\varepsilon\right\}$. We will show that $\mathcal{F}_{k}=X$. Assume on the contrary that there exists $y \in X$ such that $S_{1} y, \ldots, S_{k} y$ are linearly independent. Then the vectors $S_{1}\left(y+\alpha x_{0}\right), \ldots, S_{k}\left(y+\alpha x_{0}\right)$ are linearly independent for all but finitely many scalars $\alpha$. Hence, $S_{1}\left(x_{0}+(1 / \alpha) y\right), \ldots, S_{k}\left(x_{0}+(1 / \alpha) y\right)$ are linearly independent for all but finitely many nonzero scalars $\alpha$. This is impossible since $\mathcal{F}_{k}$ contains $\left\{x \in X:\left\|x-x_{0}\right\|<\varepsilon\right\}$. Hence, we have a desired relation $\mathcal{F}_{k}=X$.

So, the linear span of $\left\{S_{1}, \ldots, S_{k}\right\}$ is a locally linearly dependent space. We already know that then $\left\{S_{1}, \ldots, S_{k}\right\}_{F}$ is nontrivial and locally linearly dependent [5, Proposition 2.3]. This completes the proof.

\section{Nonlinear locally linearly dependent sets of operators.}

The methods of the previous section can be applied to some nonlinear sets of operators that are locally linearly dependent. We will only consider the finite-dimensional case, so operators will be identified with matrices. Let $p, q, n$ be positive integers and $W=\mathbb{F}^{n}$ the $n$-dimensional coordinate space. Let $x=\left(x_{1}, \ldots, x_{n}\right)$ be a vector of variables and suppose $S(x)=\left(s_{i j}(x)\right)$ is a $p \times q$ matrix of homogenous polynomials $s_{i j}(x) \in \mathbb{F}\left[x_{1}, \ldots, x_{n}\right]$ of fixed degree $d$. The set $\mathcal{S}=\{S(a): a \in W\}$ is called an $(n, d)$-family in $M_{p \times q}(\mathbb{F})$. $\mathcal{S}$ is locally linearly dependent if for any $u \in \mathbb{F}^{q}$ there exists a nonzero $a \in W$ such that $S(a) u=0$.

Theorem 3.1. Suppose $\mathcal{S} \subset M_{p \times q}(\mathbb{F})$ is a locally linearly dependent $(n, d)$ family and $|\mathbb{F}| \geq d n+2$. Then either there exists an $0 \neq a \in W$ such that $\operatorname{rank} S(a) \leq n-2$ or $\operatorname{rank} S(a)=n-1$ for all $0 \neq a \in W$.

If $d=1$ then $\mathcal{S}$ is a linear space of matrices. This special case coincides with the finite-dimensional case of Theorem 2.4. The proof of the general case goes through in almost the same way. Theorem 2.2 can be similarly extended to this more general nonlinear setting.

\section{Applications.}

The aim of this section is to illustrate applicability of the results of Section 2 in algebra and functional analysis. We start by an improvment of Amitsur's result on rings satisfying generalized polynomial identities [1]. 
Theorem 4.1. Let $R$ be a primitive ring considered as a dense ring of linear transformations on a vector space $V$ over a division ring $D$ with the center $C$. Let $R$ be a $C$-algebra satisfying a nontrivial generalized polynomial identity of degree $k$ which includes $n C$-independent elements (including 1 ). Then $R$ contains a finite rank transformation and $D$ is finite over $C$ with

$$
(D: C) \leq 4^{n-1}\left(n-1+n\left[\frac{k}{2}\right]\right)^{2} .
$$

Remark. Amitsur proved this result with a weaker estimate

$$
(D: C) \leq 4^{n-1}\left(\left(\begin{array}{c}
n+1 \\
2
\end{array}\right)-1+n\left[\frac{k}{2}\right]\right)^{2} .
$$

Proof. We have proved that if $T_{1}, \ldots, T_{n}$ are locally linearly dependent operators between vector spaces over a field $\mathbb{F}$, then there exists a nontrivial linear combination of these operators of rank at most $n-1$. The same result with essentially the same proof holds true if $\mathbb{F}$ is any infinite division ring. As every finite division ring ring is commutative, this statement holds for all division rings $\mathbb{F}$. In fact, we can extend this as follows: Let $U$ and $V$ be left vector spaces over a division ring $\mathbb{F}, V_{0}$ a finite-dimensional subspace of $V$, and let linear operators $T_{1}, \ldots, T_{n}: U \rightarrow V$ be locally linearly dependent modulo $V_{0}$. Then there exist $\alpha_{1}, \ldots, \alpha_{n} \in \mathbb{F}$, not all zero, such that

$$
\operatorname{rank}\left(\alpha_{1} T_{1}+\ldots+\alpha_{n} T_{n}\right) \leq \operatorname{dim} V_{0}+n-1 .
$$

To verify this extension one simply considers locally linearly dependent operators $Q T_{1}, \ldots, Q T_{n}: U \rightarrow V / V_{0}$. Here, of course, $Q: V \rightarrow V / V_{0}$ is the quotient map.

Amitsur proved this statement with the above estimate replaced by a weaker one

$$
\operatorname{rank}\left(\alpha_{1} T_{1}+\ldots+\alpha_{n} T_{n}\right) \leq \operatorname{dim} V_{0}+\left(\begin{array}{c}
n+1 \\
2
\end{array}\right)-1 .
$$

To prove the theorem one has to follow step by step the proof of Amitsur applying our better estimate instead of the Amitsur's one.

Our next application is to give a shorter and simpler proof of the characterization of commuting pairs $d, g$ of continuous derivations of a Banach algebra such that $d g(x)$ is quasi-nilpotent for every $x \in \mathcal{A}$. An interested reader can find some remarks on the background of this problem in $[\mathbf{3}$, p. 1259].

Theorem 4.2. Let $\mathcal{A}$ be a complex Banach algebra and $d, g: \mathcal{A} \rightarrow \mathcal{A}$ be continuous derivations. If $d$ and $g$ commute, then the following are equivalent. 
(i) $d g(x)$ is quasi-nilpotent for every $x \in \mathcal{A}$.

(ii) $d g(x)^{3}$ lies in the radical of $\mathcal{A}$ for every $x \in \mathcal{A}$.

(iii) if $\pi$ is a continuous irreducible representation of $\mathcal{A}$ on a Banach space $X$, then there exist linear operators $A$ and $B$ on $X$ such that $\pi(d(x))=$ $[\pi(x), A], \pi(g(x))=[\pi(x), B], x \in \mathcal{A}, A B=B A=0$, and either $A^{2}=0$ or $B^{2}=0$, unless $\pi d=0$ or $\pi g=0$.

Proof. This result has already been proved in [3]. We will just sketch the first few steps of the proof since they are the same as in [3]. First one can observe that the only nontrivial implication is (i) $\Rightarrow$ (iii). Assume, therefore, that (i) holds and that $\pi$ is a continuous irreducible representation of $\mathcal{A}$ on a Banach space $X$. Then $\mathcal{B}=\pi(\mathcal{A}) \subset \mathcal{B}(X)$ is a dense algebra of bounded linear operators on $X$. Define $D, G: \mathcal{B} \rightarrow \mathcal{B}$ by $D(\pi(x))=\pi(d(x))$, $G(\pi(x))=\pi(g(x))$. It turns out that these maps are well-defined and that there exist a commuting pair of linear operators $A$ and $B$ on $X$ such that $D(S)=[S, A]$ and $G(S)=[S, B]$ for all $S \in \mathcal{B}$. Note that $D(S)=[S, A-\lambda]$ for any scalar operator $\lambda$, and that a derivation $c D$, where $c$ is any nonzero complex number, satisfies the same assumption as $D$. Therefore, whenever it will be suitable, we will replace $A$ by $A-\lambda$ or $c A$, and, of course, we will do the same with $B$. Let us also point out that $D$ and $G$, and consequently $A$ and $B$, appear symmetrically.

So, our basic assumption is that $D G(S)=[[S, B], A]$ is quasi-nilpotent for every $S \in \mathcal{B}$ and we have to prove that there exist scalars $\lambda$ and $\mu$ such that $(A-\lambda)(B-\mu)=0$ and either $(A-\lambda)^{2}=0$ or $(B-\mu)^{2}=0$. In $[\mathbf{3}]$ this part of the proof was long, involving tedious computations. Having new results on locally linearly dependent operators we can now present a much simpler and shorter proof. It will be broken up into a series of steps. Two of them go through in exactly the same way as in $[\mathbf{3}]$. We will include them for the sake of completness. If $f$ is a linear (not necessarily bounded) functional on $X$ and $x \in X$ we denote by $x \otimes f$ a rank one operator $(x \otimes f) z=f(z) x$, $z \in X$. Note that every rank one operator on $X$ can be written in this form.

Step 4.3. Let $Y$ be a subspace of $X$ invariant under both $A$ and $B$ and assume that the restrictions of $A$ and $B$ to $Y$ satisfy $A_{\mid Y}=B_{\mid Y}$. Then there exists a complex number $\lambda$ such that $(A-\lambda)_{\mid Y}^{2}=0$.

Proof. Suppose there exists $y \in Y$ such that $y, A y$, and $A^{2} y$ are linearly independent. Then, since $\mathcal{B}$ is a dense subalgebra of $\mathcal{B}(X)$, we can find $S \in \mathcal{B}$ such that $S y=S A y=0$ and $S A^{2} y=y$. It follows that $[[S, B], A] y=y$ contradicting the fact that $[[S, B], A]$ is quasi-nilpotent. So, for every $y \in Y$ the vectors $y, A y$, and $A^{2} y$ are linearly dependent. By Kaplansky's theorem on locally algebraic operators we have either $A_{\mid Y}=\lambda I+N$ for some scalar $\lambda$ and some square-zero operator $N$, or $A_{\mid Y}=\lambda P+\mu(I-P)$ for some scalars $\lambda, \mu, \lambda \neq \mu$, and some idempotent $P \notin\{0, I\}$. Here, $I$ denotes 
the identity operator on $Y$. In the first case the desired conclusion follows trivially. In the second case there is no loss of generality in assuming that $A_{\mid Y}=B_{\mid Y}=P$. Take a nonzero $x$ from the range of $P$ and a nonzero $y$ from the null space of $P$. Then we can find $S \in \mathcal{B}$ such that $S x=y$ and $S y=x$. Consequently, $[[S, B], A](x+y)=x+y$. This contradiction shows that the second possibility cannot occur. This completes the proof of Step 4.3.

Step 4.4. Let $Y$ be a two-dimensional subspace of $X$ invariant under both $A$ and $B$. Then there exist scalars $\lambda$ and $\mu$ such that $(A-\lambda)_{\mid Y}(B-\mu)_{\mid Y}=0$ and either $(A-\lambda)_{\mid Y}^{2}=0$ or $(B-\mu)_{\mid Y}^{2}=0$.

Proof. The conclusion follows directly if $A_{\mid Y}$ is a scalar operator. If not, then because $B_{\mid Y}$ commutes with $A_{\mid Y}$, we have $B_{\mid Y}=\alpha A_{\mid Y}+\beta I$ for some scalars $\alpha$ and $\beta$. So, we may assume that $A_{\mid Y}=B_{\mid Y}$ and complete the proof using Step 4.3.

Step 4.5. Let $Y$ be a subspace of $X$ invariant under both $A$ and $B$ and assume that $A_{\mid Y}^{2}=0$. Then there exists a scalar $\lambda$ such that $A_{\mid Y}(B-\lambda)_{\mid Y}=$ 0 .

Proof. It is easy to see that all we have to do is to prove that $A B y$ belongs to the linear span of $A y$ for every $y \in Y$. Assume that this is not true for a certain $y \in Y$. Then we can find $S \in \mathcal{B}$ satisfying $S A y=0$ and $S A B y=-y$, yielding $[[S, B], A] A y=A y$. We have $A y \neq 0$ since otherwise $A B y=B A y$ would be 0 , too. This contradiction completes the proof.

Step 4.6. Let $Y$ be a subspace of $X$ invariant under both $A$ and $B$. If $A B_{\mid Y}=0$ then either $A_{\mid Y}^{2}=0$ or $B_{\mid Y}^{2}=0$.

Proof. Assume first that there are $x, y \in Y$ such that $A^{2} x$ and $B^{2} y$ are linearly independent. Then there is an $S \in \mathcal{B}$ such that $S A^{2} x=-y, S B^{2} y=$ $-x, S A x \in Y$, and $S B y \in Y$. But then $[[S, B], A](A x+B y)=A x+B y \neq 0$ since $0 \neq A^{2} x=A(A x+B y)$. Therefore, such $x$ and $y$ cannot exist. That is, $A^{2} x$ and $B^{2} y$ are dependent for any $x, y \in Y$. If neither $A^{2}$ nor $B^{2}$ is zero on $Y$, then there is $w \in Y$ such that $A^{2} Y=B^{2} Y=\operatorname{span}\{w\}$. Note that there is $y \in Y$ such that $A^{2} y=\lambda w \neq 0$ and $B^{2} y=\mu w \neq 0$. However, picking $S \in \mathcal{B}$ such that $S w=y, S A y \in Y$, and $S B y \in Y$, we arrive at $[[S, B], A]^{2} A y=\lambda \mu A y$. With this contradiction the proof of this step is completed.

Step 4.7. Let $Y$ be a subspace of $X$ invariant under both $A$ and $B$. If $A B_{\mid Y}=I$ then there exist a nonzero scalar $\lambda$ and a square-zero operator $M: Y \rightarrow Y$ such that $A_{\mid Y}=\lambda I+M$ and $B_{\mid Y}=\frac{1}{\lambda} I-\frac{1}{\lambda^{2}} M$. Here, $I$ denotes the identity operator on $Y$.

Proof. If there exists $y \in Y$ such that $y, A y$, and $A^{2} y$ are linearly independent then we can find $S \in \mathcal{B}$ such that $S y=S A^{2} y=0$ and $S A y=A y$. 
It follows that $[[S, B], A] A y=2 A y$, a contradiction. By Kaplansky's theorem on locally algebraic operators $A_{\mid Y}$ is algebraic of degree at most two. If $A_{\mid Y}=\lambda I+M$ with $M$ a square-zero (possibly zero) operator, then the desired conclusion follows directly from the equation $A B_{\mid Y}=I$. In the remaining case we have $A=\alpha P+\beta(I-P)$ for some scalars $\alpha \neq \beta, \alpha, \beta \neq 0$, and some idempotent $P: Y \rightarrow Y, P \neq 0, I$. Then $B=\alpha^{-1} P+\beta^{-1}(I-P)$ and we can find linearly independent vectors $x$ and $y$ belonging to the range of $P$ and $I-P$, respectively, and $S \in \mathcal{B}$ such that $S x=y$ and $S y=x$. It follows that $x+y$ belongs to the eigenspace of $[[S, B], A]$ corresponding to the nonzero eigenvalue $\left(2-\frac{\alpha^{2}+\beta^{2}}{\alpha \beta}\right)$. This contradiction completes the proof of this step.

Step 4.8. Assume that the operators $A, B$, and $I$ are locally linearly dependent. Then there exist scalars $\lambda$ and $\mu$ such that $(A-\lambda)(B-\mu)=0$ and either $(A-\lambda)^{2}=0$ or $(B-\mu)^{2}=0$.

Proof. If the dimension of the linear span of $A, B, I$ is 1 or 2 then we can assume with no loss of generality that either $A=B$, or $A=0$, or $B=0$. So, in this case we are done by Step 4.3. Step 4.4 gives the desired conclusion if $\operatorname{dim} X \leq 2$. It remains to consider the case that $A, B, I$ are linearly independent and $\operatorname{dim} X>2$. Then, by Theorem 2.4 there exist scalars $\alpha, \beta, \gamma$ such that $\alpha A+\beta B+\gamma I=F$ is of rank one. Note that we can assume with no loss of generality that $\gamma=0$.

Assume first that one of scalars $\alpha, \beta$, say $\alpha$, is zero. Then $B$ is of rank one. If it is square-zero, then we complete the proof using Step 4.5. If not, then after multiplying by an approprite constant, we can assume that $B$ is an idempotent of rank one. As $A$ commutes with $B$ it leaves the null space and the range of $B$ invariant. Hence, the one-dimensional range of $B$ is contained in an eigenspace of $A$ corresponding to an eigenvalue $\lambda$. It follows that $(A-\lambda) B=0$, and hence, the desired conclusion follows from Step 4.6.

It remains to consider the case that both $\alpha$ and $\beta$ are nonzero. In this case we may assume that $A=B+z \otimes f$ for some $z \in X$ and some linear functional $f$ on $X$. The null space of $f$ is invariant for both $A$ and $B$ because $A$ and $B$ commute. The restrictions of $A$ and $B$ to this common invariant subspace coincide, and so, by Step 4.3, we may assume that both these restrictions are square-zero. Let $u \in X$ be a vector such that $X$ is a direct sum of the null space of $f$ and the linear span of $u$ and define a linear operator $T: X \rightarrow X$ such that $T u=0$ and the restriction of $T$ to the null space of $f$ coincides with the restriction of $A$ to this subspace. Then $T^{2}=0$, the range of $T$ is contained in the null space of $f$, and $A=T+x \otimes f$ and $B=T+y \otimes f$ for some $x, y \in X$. Applying $A B=B A$ we get

$$
T y+f(y) x=T x+f(x) y .
$$


Multiplying by $T$ we arrive at

$$
f(y) T x=f(x) T y .
$$

We will consider three cases. If $f(x)=f(y)=0$, then clearly $A^{2} B=$ $A B^{2}=0$. In case that there was $x \in X$ such that $A B x \neq 0$ we would have $S \in \mathcal{B}$ satisfying $S A B x=x$, which would further imply $[[S, B] A] A B x=$ $A B x$, a contradiction. Therefore, $A B=0$ in this case and one can complete the proof using Step 4.6.

If $f(x)=0$ and $f(y) \neq 0$ then $T x=0$ which yields $A B=0$ and we are done.

In the remaining case that both $f(x)$ and $f(y)$ are nonzero, we may assume with no loss of generality that $f(x)=1$. From the above equations we get $T y=f(y) T x$, and consequently, $(f(y)-1) T x=y-f(y) x$. Now, if $f(y)=1$ then $T x=T y$, and consequently, $x=y$. Thus, $A=B$, and the desired conclusion follows from Step 4.3. So, assume that $f(y) \neq 1$. Then $T x$, and hence $T y$, belong to the linear span of $x$ and $y$, which is then obviously invariant under both $A$ and $B$. Let us first consider the case that $T x$ is nonzero. Since $T^{2}=0$, the vectors $x$ and $T x$ are linearly independent. Thus, the linear span of $x$ and $y$ is the same as the linear span of $x$ and $T x$. Since $A T x=B T x=0$, Step 4.4 implies that the restriction of $A^{2}$ or $B^{2}$, say $A^{2}$, to the linear span of $x$ and $y$ is zero. We already know that the restriction of $A^{2}$ to the null space of $f$ is zero. So, $A^{2}=0$ and we complete the proof in this case using Step 4.5. We will show that the remaining case, that $T x=0$ and hence $T y=0$, cannot occur. Namely, in this case $x$ and $y$ would be linearly dependent. The operators $A$ and $B$ would have matrix representations

$$
A=\left[\begin{array}{cc}
1 & 0 \\
0 & A^{\prime}
\end{array}\right] \text { and } B=\left[\begin{array}{cc}
\alpha & 0 \\
0 & A^{\prime}
\end{array}\right]
$$

with respect to the decomposition of $X$ into the direct sum of the linear span of $x$ and the null space of $f$. Here, $A^{\prime}$ is the restriction of $A$ to the null space of $f$ and $\alpha$ is a nonzero scalar. Take any nonzero $z$ in the null space of $f$ such that $A^{\prime} z=0$. Then the linear span of $x$ and $z$ is invariant under both $A$ and $B$. The restrictions of $(A-\lambda)^{2}$ and $(B-\mu)^{2}$ to this two-dimensional subspace are both nonzero for any choice of scalars $\lambda$ and $\mu$. This contradiction with Step 4.4 completes the proof.

Step 4.9. If $A B=N$ is a square-zero operator then there exist scalars $\lambda$ and $\mu$ such that $(A-\lambda)(B-\mu)=0$ and either $(A-\lambda)^{2}=0$ or $(B-\mu)^{2}=0$.

Proof. The null space of $N$ is invariant under both $A$ and $B$ and the restriction of $A B$ to this subspace is the zero operator. So, by Step 4.6 one of the restrictions of $A$ or $B$ to the null space of $N$, say the restriction of $A$, is square-zero. It follows that $A^{3} B=A^{2}(A B)=0$. Next we will see that $A^{2} B=0$. If not, then we would have an $x \in X$ with $A^{2} B x \neq 0$ 
and $S \in \mathcal{B}$ such that $S A^{2} B x=A x$. But then $[[S, B], A] A^{2} B x=A^{2} B x$, a contradiction. The next step is to prove that there is a scalar $\lambda$ such that $A B=\lambda A B^{2}$. To this end it is enough to show that $A B x$ belongs to the linear span of $A B^{2} x$ for every $x \in X$. Assume this is not true for a given $x \in X$. Then we can find $S \in \mathcal{B}$ such that $S A B x=x$ and $S A B^{2} x=0$, which further yields $[[S, B], A] A B x=A B x$, a contradiction. Now, let $x$ be any vector from $X$. Then $x=\lambda B x+(x-\lambda B x)$. We already know that $A^{2} B x=0$. Also, $x-\lambda B x$ belongs to the null space of $N$. The restriction of $A$ to this subspace is square-zero. So, $A^{2} x=0$. Applying Step 4.5 we complete the proof.

Now we are ready for the last step of the proof.

Step 4.10. If $A, B$, and $I$ are not locally linearly dependent then there exist scalars $\lambda$ and $\mu$ such that $(A-\lambda)(B-\mu)=0$ and either $(A-\lambda)^{2}=0$ or $(B-\mu)^{2}=0$.

Proof. The operators $A B, A, B$, and $I$ are locally linearly dependent. Indeed, if this were not true then there would exist $x \in X$ and $S \in \mathcal{B}$ such that $S A B x=x$ and $S A x=S B x=S x=0$, and so, $[[S, B], A] x=x$. By Theorem 2.6 and a remark following it we know that there exist scalars $\alpha$, $\beta$, and $\gamma$ such that $A B+\alpha A+\beta B+\gamma=M$ is a square-zero operator of finite rank. Replacing $A$ by $A+\beta$ and $B$ by $B+\alpha$, and then multiplying $A$ by an appropriate constant, we get $A B=N$ or $A B=I+N$ with $N$ being square-zero with finite rank. In the first case we complete the proof applying the previous step. So, assume that $A B=I+N$. Let $N$ have a matrix representation

$$
N=\left[\begin{array}{lll}
0 & 0 & 0 \\
I & 0 & 0 \\
0 & 0 & 0
\end{array}\right]
$$

with respect to a direct sum decomposition of $X$ into two appropriate finitedimensional spaces $X_{1}, X_{2}$, and a subspace $X_{3}$. The null space of $N$ is invariant for $A$ and $B$ and the restriction of $A B$ to this subspace is the identity operator. Applying Step 4.7 we can assume, after multiplying $A$ and $B$ by appropriate constants, that the restriction of $A$ to this subspace is a sum of the identity and a square-zero operator, while $B$ is a difference of these two operators. Since $A$ commutes with $N$ we have

$$
A=\left[\begin{array}{ccc}
A_{1} & 0 & 0 \\
A_{2} & A_{1} & A_{3} \\
A_{4} & 0 & A_{5}
\end{array}\right] .
$$

But

$$
\left[\begin{array}{cc}
A_{1} & A_{3} \\
0 & A_{5}
\end{array}\right]
$$


is a sum of the identity and a square-zero operator. It follows that $A_{1}$ is a sum of the identity and a square-zero operator, too. Consequently, $(A-I)^{4}=0$, and similarly, $(B-I)^{4}=0$.

So, we may assume that $A^{4}=B^{4}=0$. We will first prove that for every $x \in X$ the vector $B A^{3} x$ belongs to the linear span of $A^{3} x$. Indeed, if this was not true for some $x$ then we would have $S \in \mathcal{B}$ such that $S B A^{3} x=-A^{2} x$ and $S A^{3} x=0$ implying that $[[S, B], A] A^{3} x=A^{3} x \neq 0$. So, $B A^{3}=\lambda A^{3}$ for some scalar $\lambda$. If $A^{3} \neq 0$ then, since $B$ is nilpotent, we necessarily have $\lambda=0$. So, in this case $B A^{3}=0$. But, of course, this is true also in the case that $A^{3}=0$. We will next prove that for every $x \in X$ the vector $B^{2} A^{2} x$ belongs to the linear span of $B A^{2} x$. Indeed, if this was not true for some $x$ then we would have $S \in \mathcal{B}$ such that $S B^{2} A^{2} x=-A B x$ and $S B A^{2} x=0$ implying that $[[S, B], A] B A^{2} x=B A^{2} x \neq 0$. As before we conclude that $B^{2} A^{2}=0$ and we complete the proof using the previous step.

Acknowledgement. The authors thank Kostia Beidar for the correspondence concerning the application of our results in the theory of rings satisfying generalized polynomial identities.

\section{References}

[1] S.A. Amitsur, Generalized polynomial identities and pivotal monomials, Trans. Amer. Math. Soc., 114 (1965), 210-226, MR 30 \#3117, Zbl 0131.03202.

[2] B. Aupetit, A Primer on Spectral Theory, Springer-Verlag, 1991, MR 92c:46001, Zbl 0715.46023.

[3] M. Brešar and P. Šemrl, On locally linearly dependent operators and derivations, Trans. Amer. Math. Soc., 351 (1999), 1257-1275, MR 99e:47039, Zbl 0920.15009.

[4] I. Kaplansky, Infinite Abelian Groups, Ann Arbor, 1954, MR 16,444g, Zbl 0057.01901.

[5] D.R. Larson, Reflexivity, algebraic reflexivity and linear interpolation, Amer. J. Math., 283 (1988), 283-299, MR 89d:47096, Zbl 0654.47023.

[6] R. Lidl and H. Niderreiter, Finite Fields, Encyclopedia Math. Appl., 20, Cambridge Univ. Press, Cambridge, 1983, MR 86c:11106, Zbl 0554.12010.

[7] V. Müller, Kaplansky's theorem and Banach PI-algebras, Pacific J. Math., 141 (1990), 355-361, MR 91k:46048, Zbl 0736.46044.

Received June 23, 2000. This paper was supported in part by a grant from the Ministry of Science of Slovenia and by the Israel Science Foundation. 
E-mail address: meshulam@math.technion.ac.il

Department of Mathematics

UNIVERSITY OF LJUBLJANA

JADRANSKA 19

SI-1000 LJUBLJANA

SLOVENIA

E-mail address: peter.semrl@fmf.uni-lj.si 